\title{
A Kinematic Model for Surface Irrigation: An Extension
}

\author{
BERNARD SHERMAN \\ Department of Mathematics, New Mexico Institute of Mining and Technology, Socorro, New Mexico 87801 \\ VIJAY P. SINGH \\ Department of Civil Engineering, Louisiana State University, Baton Rouge, Louisiana 70803

\begin{abstract}
The kinematic model for surface irrigation, reported previously by Sherman and Singh (1978), is extended. Depending upon the duration of irrigation and time variability of infiltration, three cases are distinguished. Explicit solutions are obtained when infiltration is constant. When infiltration is varying in time, a numerical procedure is developed which is stable and has fast convergence. A rigorous theoretical justification is developed for computation of the depth of water at and the time history of the front wall of water advancing down an infiltrating plane or channel. A derivation is given of the
\end{abstract} \\ continuity and momentum equations when there is lateral inflow and infiltration into the channel bed.
}

\section{INTRODUCTION}

In recent years several studies have utilized a one-dimensional form of the St. Venant equations or their approximations in hydraulic modeling of surface irrigation or some aspects thereof [Bassett and Fitzsimmons, 1976; Katopodes and Strelkoff, 1977a, b; Strelkoff and Katopodes, 1977a, b; Sherman and Singh, 1978; Clemmens, 1979, 1980; Clemmens and Strelkoff, 1979; Fangmeier and Strelkoff, 1979; Chen, 1980; Fonken et al., 1980]. Sherman and Singh [1978] showed, using kinematic wave theory, that the governing equations for surface irrigation constitute a free boundary problem. A free boundary problem will also arise if the governing equations are based on the zero inertia approximation or the full form of the St. Venant equations. An explicit discussion of these mathematical issues does not occur in the other references cited above.

In irrigation hydraulics both advance and recession are free boundaries which are not known a priori but must be determined concurrently with the solution of the governing equations. Bassett [1972] and Bassett and Fitzsimmons [1976] presented a hydrodynamic model for border irrigation. They solved the St. Venant equations numerically using the method of characteristics [Streeter and Wylie, 1967]. From their discussion an explicit formulation of the free boundary problem is not clear. This also holds for the studies by Katopodes and Strelkoff $[1977 a, b]$.

Strelkoff pioneered the concept of zero-inertia in hydraulic modeling of surface irrigation [Strelkoff and Katopodes, $1977 a, b ;$ Clemmens and Strelkoff, 1979; Fangemeier and Strelkoff, 1979]. Numerical solutions were utilized, but an explicit formulation of the free boundary problem was not given. Therefore even for simple cases it is not clear what the analytical solutions will look like. The same is true of other studies on hydraulic modeling of surface irrigation cited earlier. This discussion is not intended to detract from the basic theme of these papers. These authors have made fundamental contributions to hydraulics of farm irrigation

Copyright 1982 by the American Geophysical Union.

Paper number 2 W0199.

0043-1397/82/002W-0199\$05.00 and have presented a perspective which is essential for understanding the limitations of existing techniques.

In this study we will address ourselves to the above mathematical issues, that is, (1) formulation of free boundary problems using kinematic wave theory and full dynamic equations and (2) solution of the free boundary problem using kinematic equations. Sherman and Singh [1978] developed a kinematic model for surface irrigation which considered advance, storage depletion, and recession phases of the irrigation cycle [Bassett and $\mathrm{McCool}, 1973]$. We will continue the analysis of this model here. We refer to that work for the background information but briefly outline below the formulation of the problem.

Surface irrigation essentially deals with water flowing down a plane (border) or channel and infiltrating into the channel bed. We consider a rectangular channel of uniform cross section which is initially dry. Let $x$ be the distance along the channel which extends indefinitely to the right of $x$ $=0 ; x=0$ is the position of its head. At time $t=0$, water is released at the head $x=0$. The depth of water inflow at $x=0$ is assumed to be a known time-dependent function $g(t)$. The inflow of water at $x=0$ lasts for a specified length of time $T$. When water is released, according to the discussion by Sherman and Singh [1978], there is a front wall of water which advances down the channel. This front wall of water is the advance front, that is, the interface between the water covered and uncovered part of the channel. Let $x=s(t)$ or, inversely, $t=\zeta(x)$, be the time history of that advancing front; this time history is the advance function. This front is a free boundary which has to be determined along with the depth $h(x, t)$ and velocity $u(x, t)$. Let $f(\tau)$ be the infiltration rate (volume per unit area per unit time) at time $\tau=t-\zeta(x)$; $\tau$ denotes the infiltration opportunity time at a point $x$ in the plane, that is, the interval of time that water has covered the point $x$, where $t$ is the total time elapsed since the inflow began. The infiltration rate $f(\tau)$ is assumed to depend only on the difference $\tau$ between the total elapsed time and the advance time; that is, it is time dependent but independent of $x$ for $x>0$.

The depth of water $h(x, t)$ and the unknown time history $\zeta(x)$ are subject to the following kinematic formulation: 


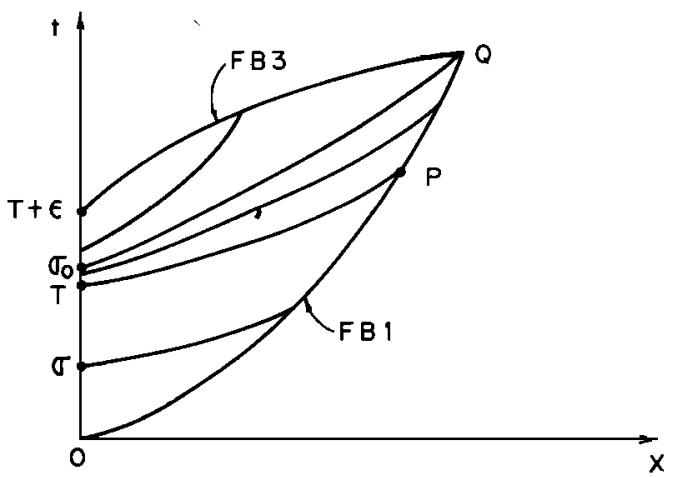

Fig. 1. Solution domain for (3).

$$
\begin{array}{cc}
\frac{\partial h}{\partial t}+\frac{\partial}{\partial x}\left[\alpha(x) h^{n}\right]=-f(t-\zeta(x)) & h(0, t)=g(t) \\
\zeta^{\prime}(x)=\left[\alpha(x) h^{n-1}(x, \zeta(x))\right]^{-1} & \zeta(0)=0
\end{array}
$$

where $\alpha(x)$ is kinematic resistence parameter, and can be determined using standard open channel flow formula. For example, if Chezy's relation is used, then $\alpha=C S^{1 / 2}$, in which $C$ is Chezy's roughness factor and $S$ is ground slope. In this case $n=1.5$. If Manning's equation is used, then $\alpha=$ $S^{1 / 2} / n_{m}$, in which $n_{m}$ is Manning's roughness factor. Here $n=$ $\frac{5}{3}$. In these two examples, $\alpha(x)$ is a constant. We assume that $g^{\prime}(t) \leqq 0$ in order to avoid shock formation. We assume that $n>1$.

Depending upon the variability of infiltration $f$ and the kinematic wave friction parameter $\alpha$, three cases were distinguished by Sherman and Singh [1980]: (1) $f$ and $\alpha$ were constant, (2) $\alpha$ was stationary but space dependent and $f$ was constant, and (3) $f$ was time variant but space independent and $\alpha$ was constant. Explicit solutions were obtained when $f$ was constant, and an approach was suggested when $f$ was time dependent. One of the assumptions imposed on the depth of inflow was $g(t)=g>0,0 \leqq t \leqq T, g(t)=0, t>$ $T$. A closer inspection of the above solutions shows that it was tacitly assumed that $T \geqq(n-1) g / f$ (see figures 4 and 8 of Sherman and Singh [1978]). In this study we relax this assumption and discuss the case where $T<(n-1) g / f$.

The case $f(\tau)$ not constant is briefly discussed by Sherman and Singh [1978] but the discussion is incorrect from (39) forward. In (39), $\psi(\xi)$ in the first equation should be $\psi(x)$. In this paper we discuss this problem again (this time correctly) and show how to obtain the solution by an iterative procedure which can be carried out on a computer. We present the results of several such calculations.

In the last section of the paper we give a derivation of the St. Venant equations when there is lateral inflow (rainfall) and infiltration. These two terms do not appear symmetrically in the momentum equation, because in lateral inflow there is no momentum contribution to the flow in the channel direction, while momentum is lost in infiltration. Versions of the momentum equation which we believe are incorrect have appeared in the literature; we hope the discussion in this paper will rectify this matter. There is a derivation by Stoker [1957, chapter 11], but infiltration is omitted in that discussion. There are a number of errors in the work by Sherman and Singh [1978] which are corrected in the companion to this paper [Sherman and Singh, this issue].

$$
\text { The Case } T<(n-1) g / f
$$

We make the additional assumptions $g(t)=g, 0 \leqq t \leqq T$, $g(t)=0, t>T, \alpha(x)=\alpha$, and $f(\tau)=f$, where $g, \alpha$, and $f$ are positive constants. The assumption that the depth of inflow $g$ at the upstream end is constant is reasonable and has been used before. However, this assumption is not necessary for obtaining numerical solutions. Although $\alpha$ varies in both $x$ and $t$, for practical considerations it is assumed to be constant. The assumption that infiltration $f$ is constant is perhaps the most restrictive. However, it is essential for obtaining explicit solutions, which can give a great deal of insight in understanding of irrigation phenomenon. Moreover, this assumption may not be unduly restrictive in modeling recession [Wu, 1972; Singh and McCann, 1979]. Again, this assumption is not required for obtaining numerical solutions. The case $T>(n-1) g / f$ has been discussed by Sherman and Singh [1978, Figure 8]. The partial differential equation in (1) is

$$
\frac{\partial h}{\partial t}+n \alpha h^{n-1} \frac{\partial h}{\partial x}=-f \quad h(0, t)=g(t)
$$

Let the continuous function $g(t, \varepsilon)$ be defined as follows: $g(t$, $\varepsilon)=g, 0 \leqq t \leqq T, g(t, \varepsilon)$ is a decreasing function in $T \leqq t \leqq T$ $+\varepsilon$ such that $g^{\prime}(t, \varepsilon) \rightarrow-\infty$ as $\varepsilon \rightarrow 0$ (e.g., $g(t, \varepsilon)=g(T+\varepsilon$ $-t) / \varepsilon$ ), and $g(t, \varepsilon)=0 ; t \geqq T$. The solution $h(x, t, \varepsilon)$ of

$$
\frac{\partial h}{\partial t}+n \alpha h^{n-1} \frac{\partial h}{\partial x}=-f \quad h(0, t, \varepsilon)=g(t, \varepsilon)
$$

tends to the solution of (2) as $\varepsilon \rightarrow 0$. Following the discussion by Sherman and Singh [1978] the characteristic curve $x(t, \sigma$, $\varepsilon) h(t, \sigma, \varepsilon)$ of (3) passing through the point $(0, \sigma, g(\sigma, \varepsilon))$ of $(x, t, h)$ space is

$$
\begin{aligned}
& h(t, \sigma, \varepsilon)=g(\sigma, \varepsilon)-f(t-\sigma) \\
& x(t, \sigma, \varepsilon)=\frac{\alpha}{f}\left\{g^{n}(\sigma, \varepsilon)-[g(\sigma, \varepsilon)-f(t-\sigma)]^{n}\right\}
\end{aligned}
$$

Referring to Figure 1, the curve OPQ (= FB1) is the free boundary $t=\zeta(x, \varepsilon)$; the curve FB3 joining $t=T+\varepsilon$ on the $t$ axis to $Q$ is the locus of points along which $h(t, \sigma, \varepsilon)$ is 0 , and curves originating on the $t$ axis (at $t=\sigma$ ) and terminating on FB1 or FB3 are $x=x(t, \sigma, \varepsilon)$. If we let $\varepsilon \rightarrow 0$, then we get, from Figure 1, Figure 2. In Figure 2 the curves $x=x(t, \sigma)$ in $D_{2}$ originate at the point $t=T$ on the $t$ axis and terminate on PQ or FB3. Physically, FB1 is the advance function and FB3

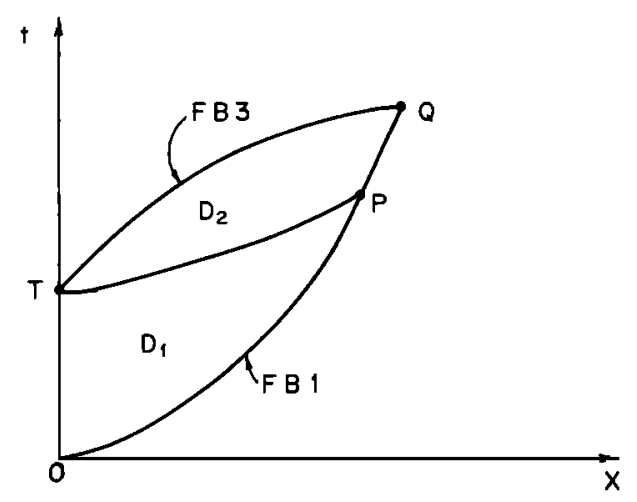

Fig. 2. Solution domain for (2). 
the recession function. The kinematic hypothesis does not accommodate vertical recession. Therefore by recession here we mean the horizontal recession. Following the discussion by Sherman and Singh [1978] we get, for $h(x, t)$ in $D_{1}$,

$$
h(x, t)=\left(g^{n}-\frac{f x}{\alpha}\right)^{1 / n}
$$

From (5) it is clear that the flow depends only on $x$ and is therefore steady but nonuniform. In $D_{2}, h(x, t)$ is defined implicitly by

$$
x=\frac{\alpha}{f}\left\{[h+f(t-T)]^{n}-h^{n}\right\}
$$

From (6) it is clear that the flow depends on both $x$ and $t$ and is therefore both unsteady and nonuniform. The part OP of FB1, i.e., the locus $t=\zeta(x)$ (in the form $x=s(t)$ ), is

$$
x=\frac{\alpha}{f}\left[g^{n}-\left(g-\frac{f t}{n}\right)^{n}\right]
$$

and FB3 is given by

$$
x=\alpha f^{n-1}(t-T)^{n}
$$

The curve $x=x(t, T)$, joining $t=T$ on the $t$ axis to $P$, is

$$
x=\frac{\alpha}{f}\left\{g^{n}-[g-f(t-T)]^{n}\right\}
$$

It remains to determine the part PQ of FB1. In Figure 1, PQ is given parametrically by [Sherman and Singh, 1978, p. 360]

$$
\begin{aligned}
\xi(\sigma, \varepsilon)= & \frac{\alpha}{f}\left\{g^{n}(\sigma, \varepsilon)-[g(\sigma, \varepsilon)-f(\eta(\sigma, \varepsilon)-\sigma)]^{n}\right\} \\
\eta^{\prime}(\sigma, \varepsilon)= & \frac{n\left(g^{\prime}(\sigma, \varepsilon)+f\right)}{(n-1) f} \\
& -\frac{n g^{\prime}(\sigma, \varepsilon) g^{n-1}(\sigma, \varepsilon)}{(n-1) f[g(\sigma, \varepsilon)-f(\eta(\sigma, \varepsilon)-\sigma)]^{n-1}} \\
\eta(T)= & \frac{n T}{n-1}
\end{aligned}
$$

Here $T \leqq \sigma \leqq \sigma_{0}(\varepsilon)$, where $\sigma_{0}(\varepsilon)$ is some number less than $T$ $+\varepsilon$. In (10) we introduce a new parameter, $\gamma=g(\sigma, \varepsilon) / g$. Then $T \leqq \sigma \leqq T+\varepsilon$ implies $1 \geqq \gamma \geqq 0$. The reason for the introduction of the parameter $\gamma$ is as follows: The parameter $\sigma$ runs from $T$ to $\sigma_{0}(\varepsilon)$, where $\xi\left(\sigma_{0}(\varepsilon), \varepsilon\right)$ and $\eta\left(\sigma_{0}(\varepsilon), \varepsilon\right)$ are the $x$ and $t$ coordinates of point $Q$ in Figure 1, and then from $\sigma_{0}(\varepsilon)$ to $T+\varepsilon$. Thus the characteristics issuing from points on the $t$ axis below $t=\sigma_{0}(\varepsilon)$ terminate on PQ, and those issuing from points above $t=\sigma_{0}(\varepsilon)$ terminate on FB3. When $\sigma$ runs from $T$ to $\sigma_{0}(\varepsilon)$, the parameter $\gamma$ runs from 1 to $\gamma_{0}(\varepsilon)=$ $g\left(\sigma_{0}, \varepsilon\right) / g$, and when $\sigma$ runs from $\sigma_{0}(\varepsilon)$ to $T+\varepsilon$, the parameter $\gamma$ runs from $\gamma_{0}(\varepsilon)$ to 0 . PQ is now represented parametrically by $x=\xi(\gamma, \varepsilon) t=\eta(\gamma, \varepsilon)$, where $\xi(\gamma, \varepsilon)$ is defined by the first line in (11) and $\eta(\gamma, \varepsilon)$ is defined by the differential equation in the second line of (11) together with the initial condition in the third line of (11). Here $T \geqq \gamma \geqq$ $\gamma_{0}(\varepsilon)$. If now we let $\varepsilon \rightarrow 0$, then $\gamma_{0}(\varepsilon)$ will tend to some value $\gamma_{0}$ between 1 and 0 , which can be determined according to the discussion below (12). The advantage of this procedure is that (11) turns into (12), which eliminates all reference to both $\varepsilon$ and $g(t, \varepsilon)$, and from (12) we have the parametric representation $x=\xi(\gamma), t=\eta(\gamma)$ of PQ in Figure 2. In this parametric representation the parameter $\gamma$ runs from 1 to $\gamma_{0}$.

Since

$$
\frac{d \eta}{d \sigma}=\frac{d \eta}{d \gamma} \frac{d \gamma}{d \sigma}=\frac{d \eta}{d \gamma} \frac{g^{\prime}(\sigma)}{g}
$$

we get, multiplying the second equation of (10) by $g / g^{\prime}(\sigma)$,

$$
\begin{aligned}
\xi(g, \varepsilon)= & \frac{\alpha g^{n}}{f}\left\{\gamma^{n}-\left[\gamma-\frac{f}{g}(\eta(\gamma, \varepsilon)-\sigma)\right]^{n}\right\} \\
\eta^{\prime}(\gamma, \varepsilon)= & \frac{n g}{(n-1) f}\left(1+\frac{f}{g^{\prime}(\sigma, \varepsilon)}\right) \\
& -\frac{n g}{(n-1) f[1-(f / g \gamma)(\eta(\gamma, \varepsilon)-\sigma)]^{n-1}}
\end{aligned}
$$

$$
\eta(1)=\frac{n T}{n-1}
$$

Here $1 \geqq \gamma \geqq \gamma_{0}(\varepsilon)$, where $\gamma_{0}(\varepsilon)=g\left(\sigma_{0}, \varepsilon\right) / g$. If in (11) we let $\varepsilon \rightarrow 0$, then $g^{\prime}(\sigma, \varepsilon) \rightarrow-\infty$ and $\sigma \rightarrow T$. Also, $\gamma_{0}(\varepsilon)$ tends to some limit $\gamma_{0}$. We get

$$
\begin{aligned}
\xi(\gamma) & \left.=\frac{\alpha g^{n}}{f}\left\{\gamma^{n}-\frac{f}{g}(\eta(\gamma)-T)\right]^{n}\right\} \\
\eta^{\prime}(\gamma) & =\frac{n g}{(n-1) f}-\frac{n g}{(n-1) f[1-(f / g \gamma)(\eta(\gamma)-T)]^{n-1}} \\
\eta(1) & =\frac{n T}{n-1}
\end{aligned}
$$

(12) is valid in $1 \geqq \gamma \geqq \gamma_{0}$, and $x=\zeta(\gamma), t=\eta(\gamma)$ is the parametric representation of $\mathrm{PQ}$ in Figure 2. Now, $\gamma=1$ corresponds to $\mathrm{P}$, and $\gamma=\gamma_{0}$ corresponds to $Q ; \gamma_{0}$ is determined by the fact that $x_{0}=\xi\left(\gamma_{0}\right), t_{0}=\eta\left(\gamma_{0}\right)$ satisfies (8).

We can approximate $\eta(\gamma)$ by a power series in $\gamma-1$ to the second degree term:

$$
\eta(\gamma)=a_{0}+a_{1}(\gamma-1)+a_{2}(\gamma-1)^{2}
$$

We have

$$
\begin{aligned}
a_{0} & =\eta(1)=\frac{n T}{n-1} \quad a_{1}=\eta^{\prime}(1)=\frac{n g}{(n-1) f}\left(1-\frac{1}{\omega^{n-1}}\right) \\
a_{2} & =\frac{1}{2} \eta^{\prime \prime}(1)=\frac{n}{2 \omega^{n}}\left[\frac{n g}{(n-1) f}\left(\frac{1}{\omega^{n-1}}-1\right)+\frac{T}{n-1}\right] \\
\omega & =1-\frac{f T}{g(n-1)}
\end{aligned}
$$

Note that $0<\omega<1$. This implies that $a_{1}<0$ and $a_{2}>0$. We write (13) as

$$
t=a_{0}+a_{1}(\gamma-1)+a_{2}(\gamma-1)^{2}
$$


and solve for $\gamma$, getting

$$
\gamma=1+\frac{1}{2 a_{2}}\left\{-a_{1}-\left[a_{1}^{2}+4 a_{2}\left(t-a_{0}\right)\right]^{1 / 2}\right\}
$$

If we write the first equation of (12),

$$
x=\frac{\alpha g^{n}}{f}\left\{\gamma^{n}-\left[\gamma-\frac{f}{g}(t-T)\right]^{n}\right\}
$$

and then replace the $\gamma$ in (15) by (14), we get approximately, the equation of PQ in the form of $x$, a function of $t$. Here $t \geqq$ $a_{0}=n T /(n-1)$.

In the case $T>(n-1) g / f$, discussed by Sherman and Singh [1978], the characteristics in $D_{1}$ are given by (14) of that paper:

$$
x=\frac{\alpha}{f}\left\{g^{n}-[g-f(t-\sigma)]^{n}\right\} \quad 0 \leqq \sigma \leqq T
$$

and on this characteristic

$$
h=g-f(t-\sigma) \quad 0 \leqq \sigma \leqq T
$$

In $D_{2}$ we have

$$
\left.x=\frac{\alpha g^{n}}{f}\left\{\gamma-\frac{f}{g}(t-T)\right]^{n}\right\} \quad 0 \leqq \gamma \leqq 1
$$

and on each of these

$$
h=g \gamma-f(t-T) \quad 0 \leqq \gamma \leqq 1
$$

These same equations hold in the case $T<(n-1) g / f$. Of course, $h$ is given in $D_{1}$ and $D_{2}$ in both cases by (5) and (6).

\section{The Case $f(\tau)$ Not Constant}

We assume that $0<f(\tau) \leqq b$ and that $\alpha(x)=\alpha=$ constant. The introduction of the new variable $\tau=t-\zeta(x)$ yields, from (1), the equations for $k(x, \tau)=h(x, \tau+\zeta(x))$ :

$$
\begin{gathered}
{\left[1-n \alpha k^{n-1} \zeta^{\prime}(x)\right] k_{\tau}+n \alpha k^{n-1} k_{x}=-f(\tau) \quad k(0, \tau)=g(\tau)} \\
\zeta^{\prime}(x)=\left[\alpha k^{n-1}(x, 0)\right]^{-1} \quad \zeta(0)=0
\end{gathered}
$$

$k(x, 0)=h(x, \zeta(x))$ is the depth of the front wall of water. From (17) we get the partial differential-difference equation [(38) of Sherman and Singh, 1978]

$\left[1-n\left(\frac{k(x, \tau)}{k(x, 0)}\right)^{n-1}\right] k_{\tau}+n \alpha k^{n-1}(x, \tau) k_{x}=-f(\tau)$

$$
k(0, \tau)=g(\tau)
$$

Following the discussion by Sherman and Singh [1978], we specify that $k(x, 0)=\Psi(x), \Psi(0)=g(0)$, on the $x$ axis, and then solve

$$
\begin{array}{r}
{\left[1-n\left(\frac{k(x, \tau)}{\Psi(x)}\right)^{n-1}\right] k_{\tau}+n \alpha k^{n-1}(x, \tau) k_{x}=-f(\tau)} \\
k(x, 0)=\Psi(x)
\end{array}
$$

We wish to determine $\Psi(x)$ so that the solution $k(x, \tau)$ of (19) has the property $k(0, \tau)=g(\tau)$. Let

$$
x=x(\sigma, \xi ; \Psi) \quad \tau=\tau(\sigma ; \xi ; \Psi) \quad k=k(\sigma, \xi ; \Psi)
$$

be the characteristic of (19) passing through the point $(\xi, 0$, $\Psi(\xi))$ of $(x, \tau, k)$ space. Here $\sigma$ is the running parameter along the characteristics; if $\sigma=0$ in (20), we get

$$
x(0, \xi ; \Psi)=\xi \quad \tau(0, \xi ; \Psi)=0 \quad k(0, \xi ; \Psi)=\Psi(\xi)
$$

The characteristic equations of (19) are

$$
\frac{d x}{d \sigma}=n \alpha k^{n-1} \quad \frac{d \tau}{d \sigma}=1-n\left(\frac{k}{\Psi(x)}\right)^{n-1} \quad \frac{d k}{d \sigma}=-f(\tau)
$$

and these are subject to the initial conditions (21). The characteristics (20), taken together, constitute a surface containing the curve $\Gamma=\{x=\xi, \tau=0, k=\Psi(\xi)\}$; indeed, (20) is the parametric representation of that surface. If we can solve the first two equations of (20) for $\sigma$ and $\xi$ in terms of $x$ and $\tau$, then, by substituting in the third equation of (20), we get the solution of (19). To this end we note that the Jacobian $j(\sigma, \xi ; \Psi)=x_{\sigma} \tau_{\xi}-x_{\xi} \tau_{\sigma}$ has the value at $\sigma=0$, using (21) and (22), $j(0, \xi ; \Psi)=n-1$. Since $j(0, \xi ; \Psi) \neq 0, j(\sigma, \xi$; $\Psi) \neq 0$ in the neighborhood of $\sigma=0$. Thus there is a surface $k(x, \tau ; \Psi)$ containing the curve $\Gamma$ satisfying (19). We note that the existence of $x_{\xi}$ and $\tau_{\xi}$ is required; this will require the existence of $\Psi^{\prime}(\xi)$. If $k(0, \tau ; \Psi)$ is defined for some interval 0 $\leqq \tau \leqq \tau_{0}$, then $k(0, \tau ; \Psi)=g(\tau)$ constitutes an equation for the determination of $\Psi(x)$. The $k(x, \tau)$ corresponding to this $\Psi(x)$ satisfies (18).

We can simplify the problem by taking $x$ as the parameter. We note, from the first equation of (22), that since $k>0, x$ is an increasing function of $\sigma$. Thus we have two functions,

$$
\tau=\tau(x, \xi ; \Psi) \quad k=k(x, \xi ; \Psi)
$$

such that

$$
\frac{d \tau}{d x}=\frac{1-n(k / \Psi(x))^{n-1}}{n \alpha k^{n-1}} \quad \frac{d k}{d x}=-\frac{f(\tau)}{n \alpha k^{n-1}}
$$

subject to the initial conditions

$$
\tau(\xi, \xi ; \Psi)=0 \quad k(\xi, \xi ; \Psi)=\Psi(\xi)
$$

$\tau$ and $k$ are defined on $S\left(\xi_{0}\right)=\left\{0 \leqq x \leqq \xi, 0 \leqq \xi \leqq \xi_{0}\right\}$, where $\Psi(\xi)>0$ on $0 \leqq \xi \leqq \xi_{0}$. If $\tau_{\xi}>0$ on $S\left(\xi_{0}\right)$, then (23) determines a function $k(x, \tau ; \Psi)$, satisfying (19). We wish to determine $\Psi(x)$ so that $k(0, \tau ; \Psi)=g(\tau)$; in terms of the functions (23), this can be expressed

$$
k(0, \xi ; \Psi)=g(\tau(0, \xi ; \Psi))
$$

The solution $\Psi(x)$ of (26) determines $k(x, \tau)$, satisfying (18). We can achieve further simplifications by introducing

$$
\begin{array}{r}
F(x, \xi ; \phi)=k^{n}(x, \xi ; \Psi) \\
\phi(x)=\Psi^{n}(x) \quad \gamma(\tau)=g^{n}(\tau)
\end{array}
$$

The $\tau$ and $F$ satisfy

$$
\begin{aligned}
\frac{d \tau}{d x} & =\frac{1}{n \alpha} F^{-(n-1) / n}-\frac{1}{\alpha}[\phi(x)]^{-(n-1) / n} \\
\frac{d F}{d x} & =-\frac{1}{\alpha} f(\tau)
\end{aligned}
$$




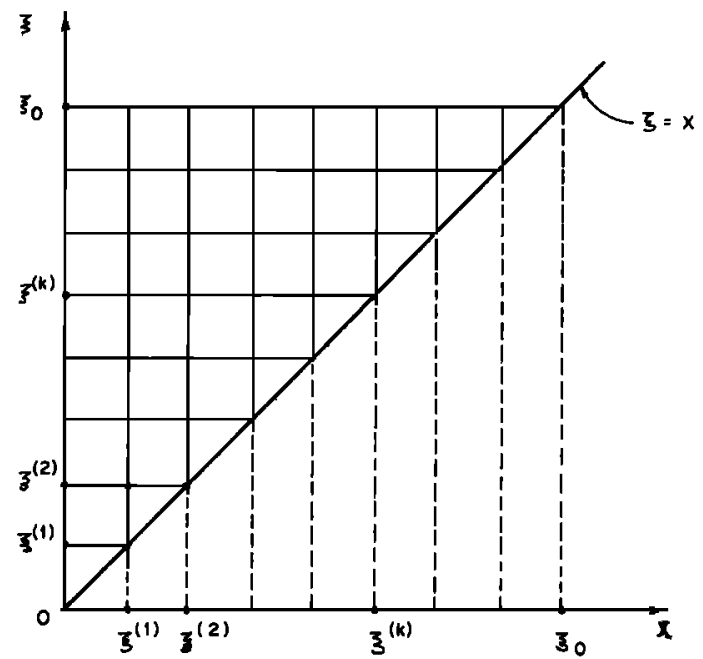

Fig. 3. Rectangular grid for numerical solution of (28)-(29) and (32).

subject to

$$
\tau(\xi, \xi ; \phi)=0 \quad F(\xi, \xi ; \phi)=\phi(\xi)
$$

(26) becomes

$$
F(0, \xi ; \phi)=\gamma(\tau(0, \xi ; \phi))
$$

We can write (28) and (29) in the following integral form (here we write $\tau(x, \xi)$ and $F(x, \xi)$, omitting $\phi)$ :

$$
\begin{aligned}
\tau(x, \xi)= & -\frac{1}{n \alpha} \int_{x}^{\xi}[F(\sigma, \xi)]^{-(n-1) / n} d \sigma \\
& +\frac{1}{\alpha} \int_{x}^{\xi}[\phi(\sigma)]^{-(n-1) / n} d \sigma
\end{aligned}
$$

$F(x, \xi)=\phi(\xi)+\frac{1}{\alpha} \int_{x}^{\xi} f(\tau(\sigma, \xi)) d \sigma$

Using the second equation of (31), we can write (30) as

$$
\phi(\xi)=-\frac{1}{\alpha} \int_{0}^{\xi} f(\tau(\sigma, \xi)) d \sigma+\gamma(\tau(0, \xi))
$$

(31) and (32) constitute three integral equations for the functions $\tau, F$, and $\phi$. The solution of (17) is equivalent to the solution of (31) and (32); if $\tau=\tau(x, \xi)$ is solved for $\xi, \xi=\xi(x$, $\tau)$, then the solution of (17) is

$$
k(x, \tau)=[F(x, \xi(x, \tau))]^{1 / n} \quad \zeta(x)=\frac{1}{\alpha} \int_{0}^{x} \frac{d \sigma}{[\phi(\sigma)]^{(n-1) / n}}
$$

The solution of (1) is given by $\zeta(x)$ in (33) and by $h(x, t)=k(x$, $t-\zeta(x))$.

The solution of (31) and (32) can be obtained explicitly in the case $f(\tau)=f=$ constant and $g(\tau)=g=$ constant. For this case and also for the case $f(\tau)=f=$ constant and $g^{\prime}(\tau)$ $<0$, we refer the reader to Sherman and Singh [1978] and Sherman [1981]. When $f(\tau)$ is not constant we use the iterative argument (34) below:

$\frac{d \tau_{k}}{d x}=\frac{1}{n \alpha} F_{k}^{-(n-1) / n}-\frac{1}{\alpha}\left[\phi_{k-1}(x)\right]^{-(n-1) / n} \quad \tau_{k}(\xi, \xi)=0$

$$
\begin{aligned}
& \frac{d F_{k}}{d x}=-\frac{1}{\alpha} f\left(\tau_{k}\right) \quad F_{k}(\xi, \xi)=\phi_{k-1}(\xi) \\
& \phi_{0}(x)=a \quad 0 \leqq x \leqq \xi_{0} \\
& \phi_{k}(\xi)=-\frac{1}{\alpha} \int_{0}^{\xi} f\left(\tau_{k}(\sigma, \xi)\right) d \sigma+\gamma\left(\tau_{k}(0, \xi)\right)
\end{aligned}
$$

Here $a$ is any number such that $0<a<\gamma(0)$ and $\xi_{0}$ is a number which can be determined once $a$ has been chosen; in the case $\gamma(t)=\gamma=$ constant, it is any number satisfying

$$
0<\xi_{0}<\min \left(\xi_{0}(a), \mathrm{H}(\mathrm{a})\right)
$$

where

$$
\begin{gathered}
\xi_{0}(a)=\frac{\alpha}{b}(\gamma-a) \\
H(a)=n \alpha[A(n+2)(n-1)]^{-1 / 2} a^{(2 n-1) / 2}
\end{gathered}
$$

Here $A$ is the maximum of $\left|f^{\prime}(\tau)\right|, \tau \geqq 0$. The right side of (35) is maximum when $a$ is selected to be the root of $\xi_{0}(a)=H(a)$. For the determination of $\xi_{0}$ when $\gamma(t)$ is not constant, we refer to Sherman [1981, p. 92-93]. We can explain the iterative process (34) in greater detail as follows: Having made a choice of $a$, we then determine $\xi_{0}$. For each $\xi, 0<\xi$ $\leqq \xi_{0}$, the two differential equations on the first two lines of (34), subject to the initial conditions indicated, determine the two functions $\tau_{1}(x, \xi)$ and $F_{1}(x, \xi)$ on $0 \leqq x \leqq \xi$. Thus $\tau_{1}$ and $F_{1}$ are determined on $S\left(\xi_{0}\right)$. The function $\tau_{1}(x, \xi)$, inserted into the right side of the last equation of (34), determines $\phi_{1}(\xi)$ on $0 \leqq \xi \leqq \xi_{0}$. We now repeat this process with $\phi_{1}(x)$, thus determining $\tau_{2}(x, \xi)$ and $F_{2}(x, \xi)$ on $S\left(\xi_{0}\right)$. The sequences of function $\phi_{k}, \tau_{k}, F_{k}$ generated in this manner converge to the solution $\phi, \tau, F$ of (31) and (32) [Sherman, 1981]. Also, $a$ $\leqq \phi_{k}(\xi) \leqq \gamma(0)$, so that $a \leqq \phi(\xi) \leqq \gamma(0)$.

We note that $\phi(x)=\Psi^{n}(x)$ and that $\Psi(x)=k(x, 0)=h(x$, $\xi(x))$ is the depth of the front wall of water. We would therefore want $\xi_{0}$ to be such that $\Psi\left(\xi_{0}\right)$ (and therefore also $\left.\phi\left(\xi_{0}\right)\right)$ to be very nearly zero. But $\phi\left(\xi_{0}\right) \geqq a>0$, so we have only a partial determination of the solution. Furthermore, if we take $a$ to be small, then (35) shows, in the case $\gamma(t)=\gamma=$ constant, that $\xi_{0}$ is also small, so that $\phi\left(\xi_{0}\right)$ is near $\gamma(0)$ rather than 0 . This situation would make impractical the iterative solution described above. Fortunately, experimentation on the computer shows that the restriction (35) in the case $\gamma(t)$ $=\gamma=$ constant, which is a sufficient condition for the convergence of the iterative procedure, is in fact not necessary. More precisely, if we take $a$ to be very small, then we may select $\xi_{0}$ to be larger than that required by (35) and still get convergence of the iterative process (34). Indeed, we may on the computer consecutively increase $\xi_{0}$ and get solutions from (34) such that $\phi\left(\xi_{0}\right)$ is sufficiently small for practical purposes. Thus (34) is a constructive and practical procedure for the solution of (1).

\section{Discussion of The Calculation AND Numerical Results}

We will now discuss the numerical experimentation on the computer. In this study our objective is not to validate the kinematic model using field observations. This constitutes a separate portion of the study, which will be reported in the near future. Here our objective is to develop a numerical 


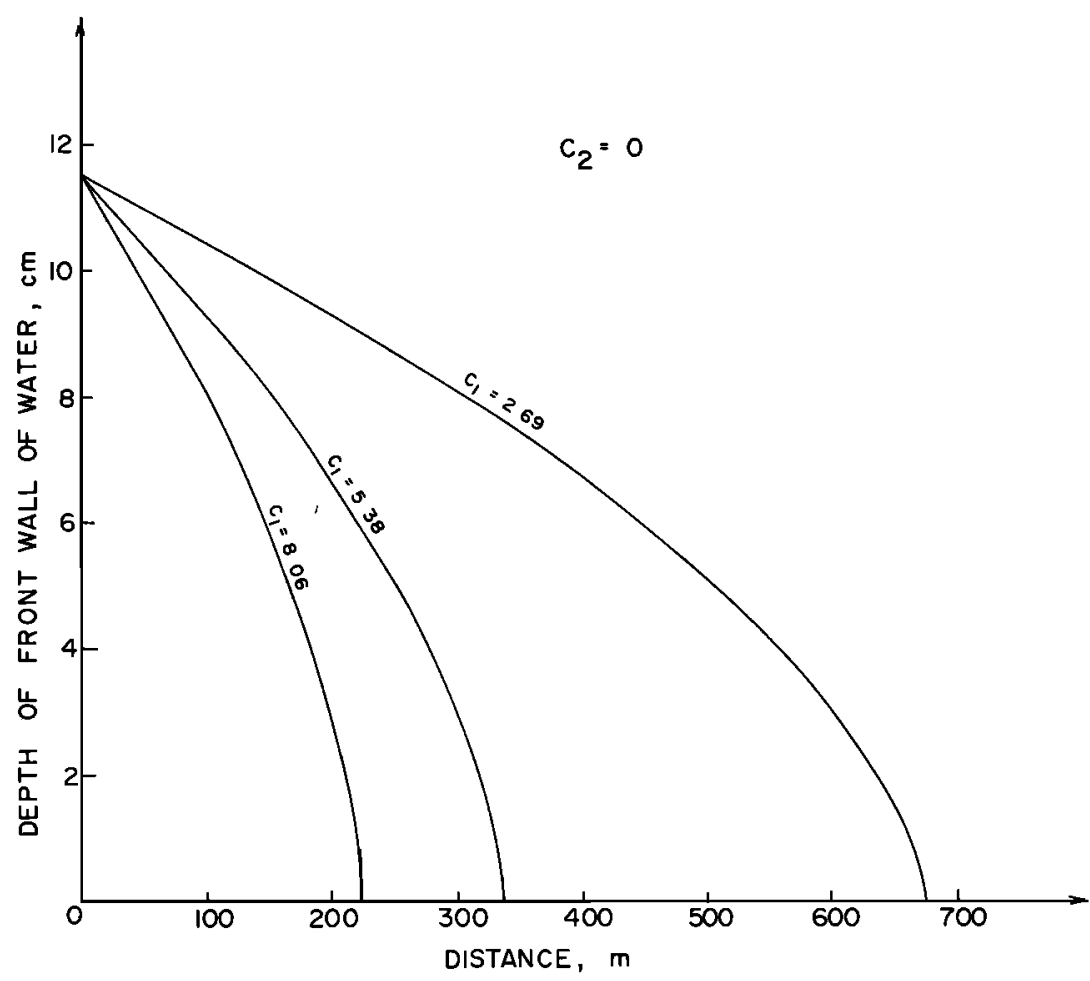

Fig. 4. The depth of front wall of water as a function of $x$ for data of Kincaid [1970].

algorithm to obtain solutions to the kinematic model under sufficiently general conditions and to determine if this algorithm has desirable mathematical properties. We consider the case $g(t)=g=$ constant only, although the procedure would also be valid for $g(t)$ not constant. We take $g=11.5$ $\mathrm{cm}$ and $f(\tau)=c_{1} e^{-c_{2} \tau}$, where $c_{1}$ and $c_{2}$ are constants. We subdivide both the $x$ and $\xi$ axes by equally spaced points 0 , $\xi^{(1)}, \xi^{(2)}, \cdots, \xi^{(m)}=\xi_{0}$, thus obtaining the grid of Figure 3 .
On this grid we implement the iterative procedure (34) as discussed above, using the fourth-order Runge-Kutta method. From the $\phi(x)$ obtained in this manner we calculate the free boundary $t=\zeta(x)$ according to the second equation of (33). For the calculation of $\phi(x)$ and $\zeta(x)$ we used six sets of $c_{1}$ and $c_{2}$ : (1) $c_{1}=2.69, c_{2}=0$, (2) $c_{1}=5.38, c_{2}=0$, (3) $c_{1}=$ $8.05, c_{2}=0$, (4) $c_{1}=2.69, c_{2}=01.4$, (5) $c_{1}=5.38, c_{2}=0.14$, and (6) $c_{1}=8.06, c_{2}=0.14$, with $c_{1}$ in $\mathrm{cm} / \mathrm{h}$ and $c_{2}$ in $1 / \mathrm{h}$.

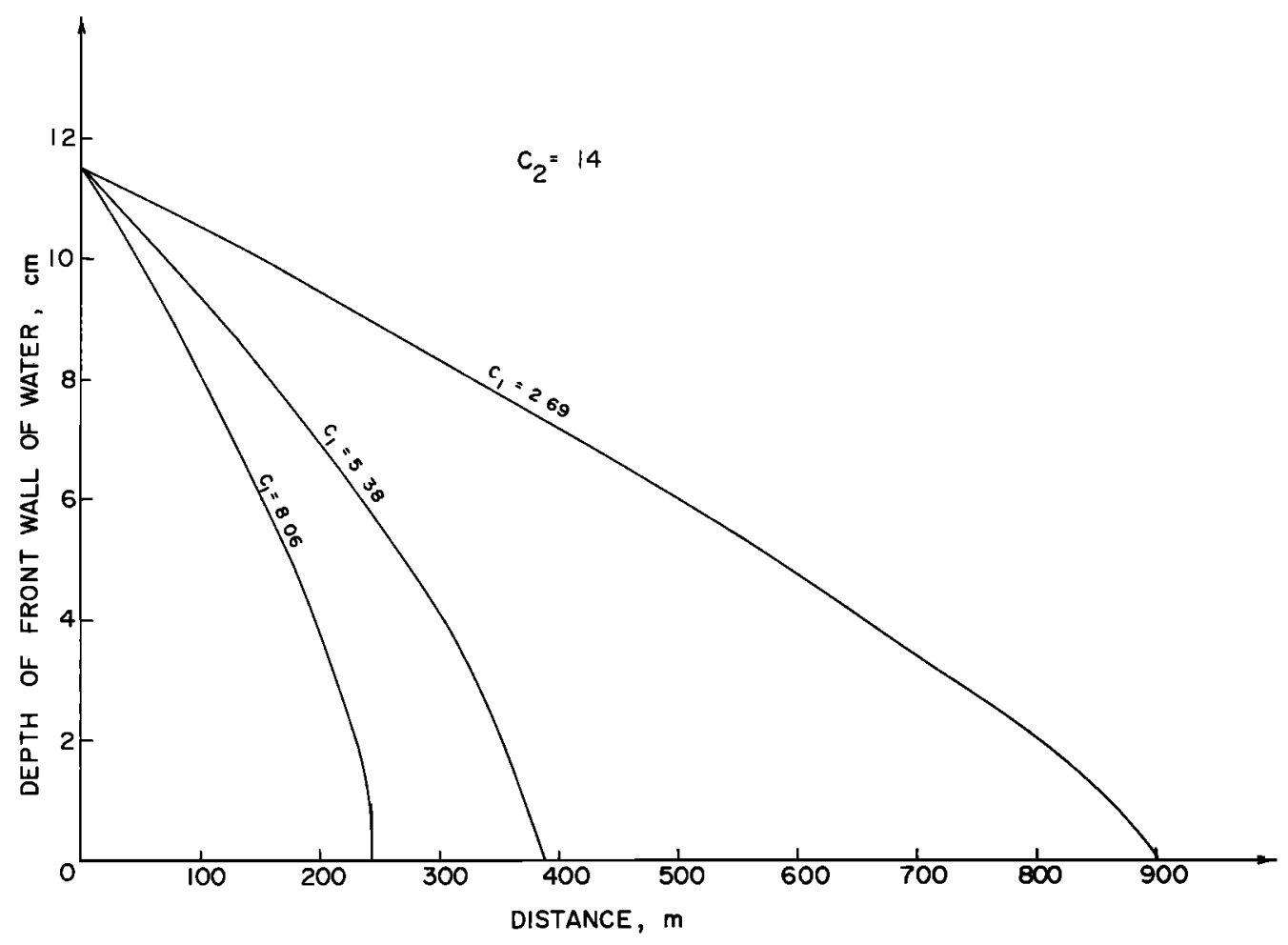

Fig. 5. The depth of front wall of water as a function of $x$ for data of Kincaid [1970]. 


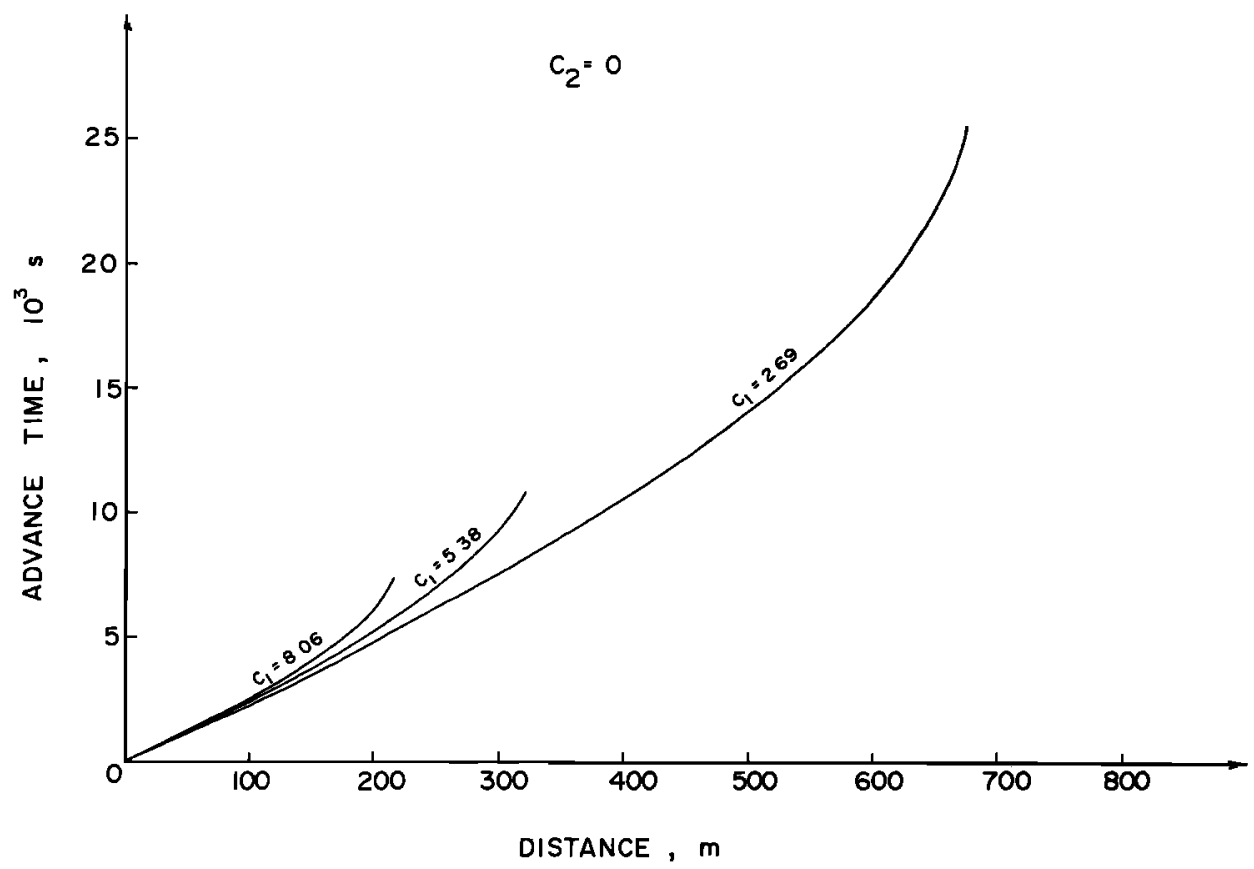

Fig. 6. Advance time as a function of $x$ for data of Kincaid [1970].

This data corresponds to border 1M of Kincaid [1970], where the slope was 0.00017 , length $19812 \mathrm{~cm}$, and width $783.4 \mathrm{~cm}$. The discharge at the upstream end of the border was 50.17 $\mathrm{cm}^{3} \mathrm{~s}^{-1} \mathrm{~cm}^{-1}$. For these values the parameter $\alpha$ was found to be 0.86 from Manning's equation; $n$ was therefore $\frac{5}{3}$.

For each set of data, $\phi(x)$ was determined as shown in Figures 4 and 5 . Figure 4 presents the case when infiltration is a constant for which we have analytical solutions. The numerical scheme was checked against this case. It was found that the numerical solutions were identical to analytical solutions. Furthermore, the numerical scheme converged very fast. As seen from the Figures 4 and 5 , the higher the infiltration rate, the lower the length for which $\phi$ becomes zero, as is physically plausible. The function $\phi(x)$ is quite sensitive to the choice of parameters in the infiltration function.

For each of the same sets of data the free boundary $t=$ $\zeta(x)$ was computed as shown in Figures 6 and 7 . We observe that the higher the infiltration, the less the distance traveled by the front wall of water. The depth of water along the free boundary is not zero except at the end of its traveled distance. The free boundary is quite sensitive to the parameters in the infiltration function. Therefore an accurate determination of infiltration parameters is crucial in irrigation flow modeling.

\section{The Continuity and Momentum Equations WITH LATERAL INFLOW AND INFILTRATION}

The continuity and momentum equations for channel flow are derived by Stoker [1957, chapter 10], when there is lateral inflow but no infiltration. In some papers the infiltration term $q$ is merely replaced by $q-f$ in these equations. While this is valid in the continuity equation, it is not valid for the momentum equation because lateral inflow and infiltration are different from the point of view of gain or loss of momentum in the direction of flow. We give here a derivation which takes this difference into account. In this derivation we make the usual assumptions of shallow water theory to the lowest order, as described by Stoker [1957]. We assume that the channel is wide and rectangular, of constant width $b$, and that the slope $S$ is constant and small. $S_{f}(u, h)$ is the friction slope, $u(x, t)$ and $h(x, t)$ the velocity and depth of water, and $q(x, t)$ and $f(x, t)$ the lateral inflow in volume per unit area per unit time. Then the continuity and momentum equations are

$$
\begin{aligned}
& h_{t}+(u h)_{x}=q-f \\
& u_{t}+u u_{x}+g h_{x}=g\left(S-S_{f}\right)-q u / h
\end{aligned}
$$

To derive the continuity equation, let $x=s_{1}(t)$ and $x=s_{2}(t)$, $s_{2}(t)>s_{1}(t)$, be plane sections moving with the water, i.e., each section contains, at all times, the same water particles. Then the mass $m(t)$ between the section is

$$
m(t)=\rho b \int_{s_{1}(t)}^{s_{2}(t)} h(x, t) d x
$$

where $\rho$ is the density. From (37) we get

$$
\begin{aligned}
m^{\prime}(t)= & \rho b\left[\int_{s_{1}(t)}^{s_{2}(t)} h_{t}(x, t) d x+h\left(s_{2}(t), t\right) s_{2}{ }^{\prime}(t)\right. \\
& \left.-h\left(s_{1}(t), t\right) s_{1}{ }^{\prime}(t)\right] \\
& -\rho b\left[\int_{s_{1}}^{s_{2}} h_{t} d x+\left(h_{2}-h_{1}\right) u_{2}+h_{1}\left(u_{2}-u_{1}\right)\right]
\end{aligned}
$$

where $h_{k}=h\left(s_{k}(t), t\right)$ and $u_{k}=u\left(s_{k}(t), t\right), k=1,2$. We have also

$$
m^{\prime}(t)=\rho b \int_{s_{1}(t)}^{s_{2}(t)}(q(x, t)-f(x, t)) d x
$$

so

$$
\begin{aligned}
\int_{s_{1}}^{s_{2}}(q-f) d x=\int_{s_{1}}^{s_{2}} h_{t} d x+\left(h_{2}-h_{1}\right) & u_{2} \\
& +h_{1}\left(u_{2}-u_{1}\right)
\end{aligned}
$$




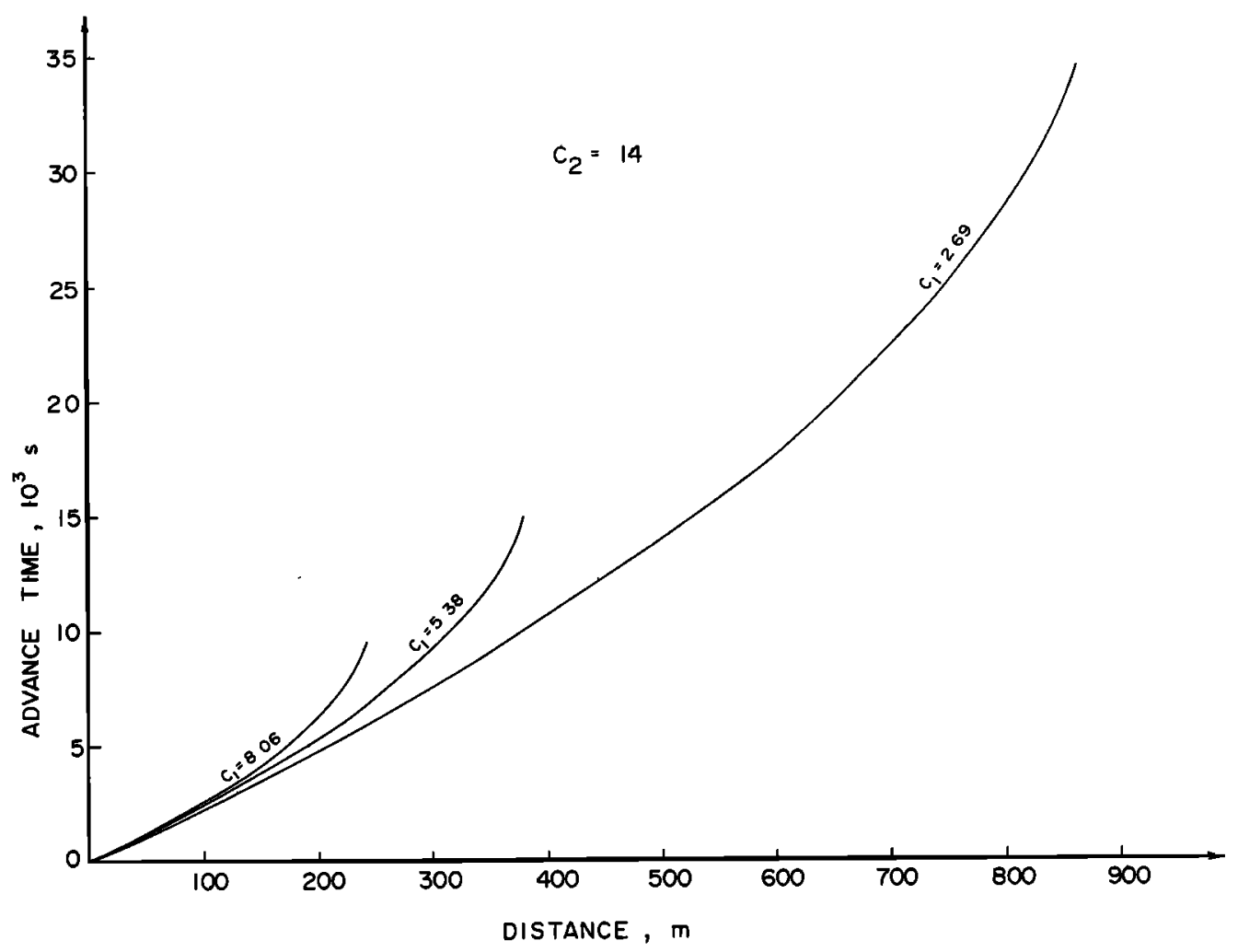

Fig. 7. Advance time as a function of $x$ for data of Kincaid [1970].

In (38) we divide by $s_{2}(t)-s_{1}(t)$ and let $s_{1}(t) \uparrow x$ and $s_{2}(t) \downarrow x$. Then we get $q-f=h_{t}+h_{x} u+h u_{x}$, which is the first equation of (36).

The momentum $M(t)$ between the two sections is

$$
M(t)=\rho b \int_{s_{1}(t)}^{s_{2}(t)} u(x, t) h(x, t) d x
$$

so that, as above,

$$
\begin{aligned}
M^{\prime}(t)=\rho b\left\{\int_{s_{1}}^{s_{2}}(u h)_{t} d x+\left[(u h)_{2}\right.\right. & \left.-(u h)_{1}\right] u_{2} \\
& \left.+(u h)_{1}\left(u_{2}-u_{1}\right)\right\}
\end{aligned}
$$

where the subscript $k$ refers to evaluation at $\left(s_{k}(t), t\right) . M^{\prime}(t)$ is the sum of three terms: the rate of momentum loss through infiltration (there is no gain or loss of momentum in the $x$ direction through lateral inflow), the body force, and the net pressure force at the two sections. The first of these terms is

$$
-\rho b \int_{s_{1}(t)}^{s_{2}(t)} f(x, t) u(x, t) d x
$$

Following Stoker [1957, pp. 454-455], and making the same small slope approximations, the body force term is, to the first order in $s_{2}(t)-s_{1}(t)$,

$$
\operatorname{\rho bgh}\left(S-S_{f}\right)\left(s_{2}(t)-s_{1}(t)\right)
$$

and the net pressure force is, to the first order in $s_{2}(t)-s_{1}(t)$,

$$
-\rho b g h h_{x}\left(s_{2}(t)-s_{1}(t)\right)
$$

From (39), (40), (41), and (42) we get, on dividing by $s_{2}(t)-$ $s_{1}(t)$ and letting $s_{1}(t) \uparrow x$ and $s_{2}(t) \downarrow x$, $(u h)_{t}+(u h)_{x} u+u h u_{x}=-f u+g h\left(S-S_{f}\right)-g h h_{x}$

From (43) we get, using the first equation of (36), the second equation of (36).

A free boundary problem for (36) can be formulated as follows: suppose $u_{0}>0$ and $h_{0}>0$ are such that $S-S_{f}\left(u_{0}\right.$, $\left.h_{0}\right)=0$; the infiltration function $f(x, t)$ is 0 for $x<0$ and is a function of $t$ only for $x>0$; the lateral input function $q(x, t)$ is 0 for all $x$ and $t$. Suppose a piston fits the channel and moves with velocity $u_{0}$ when $x<0$; to the left of the piston there is the flow regime $u=u_{0}, h=h_{0}$. We note that this flow regime satisfies (36) when $x<0$. When the piston reaches $x=0$ (at time $t=0$ ), it moves in such a manner that the height at the piston face is the prescribed function $h(x)$, where $h^{\prime}(x) \leqq 0$. This last restriction is imposed to avoid shock formation. Let $x=s(t), s(0)=0$, be the piston motion when $x \geqq 0$. It is clear that $s(t)$ is an increasing function of $x$, so it has the inverse $t$ $=\zeta(x)$. This time history $x=s(t)$ or $t=\zeta(x)$ is a free boundary. For this problem in the domain $D=\{t>0, x<$ $s(t)\}$ of the $(x, t)$ plane, we get the formulation

$$
\begin{gathered}
h_{t}+(u h)_{x}=-f(x, t-\zeta(x)) \text { in } D \\
u_{t}+u u_{x}+g h_{x}=g\left(S-S_{f}(u, h)\right) \text { in } D \\
u(x, 0)=u_{0} \quad h(x, 0)=h_{0} \quad x \leqq 0 \\
h(x, \zeta(x))=h(x) \quad u(x, \zeta(x))=\left[\zeta^{\prime}(x)\right]^{-1} \quad x>0
\end{gathered}
$$

When $h(x) \equiv 0$ and $S, S_{f}$, and $f$ are 0 , this is the dambreaking problem discussed by Stoker [1957, p. 313]. The solution of (44) may be compared with the solution of (1) if, in (1), we choose $g(t)=h(0, t)$, where $h(0, t)$ is the $h(x, t)$ of (44) evaluated at $x=0$. It is plausible that for small $S, h(0, t)$ is a decreasing function of $t$. Thus with this choice of $g(t)$ we are in position to compare the two free boundaries $t=\zeta(x)$ of 
(1) and (44), with $h(x) \equiv 0$ in (44). This comparison will provide some idea of the validity of the kinematic wave approximation, particularly in its dependence on $S$ and $C$.

Acknowledgments. This study was supported in part by funds provided by the National Science Foundation under the project Free Boundary Problems in Water Resource Engineering, NSF-ENG-7923345.

\section{REFERENCES}

Bassett, D. L., Mathematical model of water advance in border irrigation, Trans. ASAE, 15(5), 992-995, 1972.

Bassett, D. L., and D. K. McCool, A mathematical model of water advance and flow in small earth channels, project completion report, Dep. of Agric. Eng., Wash. State Univ., Pullman, 1973.

Bassett, D. L. and D. W. Fitzsimmons, Simulating overland flow in border irrigation, Trans. ASAE, 19(4), 666-671, 1976.

Chen, B. J., Numerical solutions to the kinematic model of surface irrigation, M.S. thesis, Miss. State Univ., Mississippi State, 1980.

Clemmens, A. J., Verification of the zero-inertia model for border irrigation, Trans. ASAE, 22(6), 1306-1309, 1979.

Clemmens, A. J., Depths of flow in level basins, Trans. ASAE, 23(4), 910-913, 1980

Clemmens, A. J., and T. Strelkoff, Dimensionless advance for level basin irrigation, J. Irrig. Drain. Div., Am. Soc. Civ. Eng., I05(IR3), 259-273, 1979.

Fangmeier, D. D., and T. Strelkoff, Mathematical models and border irrigation design, Trans. ASAE, 22(1), 93-99, 1979.

Fonken, D. W., T. Carmody, E. M. Laursen, and D. D. Fangmeier, Mathematical model of border irrigation, J. Irrig. Drain. Div. Am. Soc. Civ. Eng., 106(IR3), 203-220, 1980.

Katopodes, N. D., and T. Strelkoff, Hydrodynamics of border irrigation-complete model, J. Irrig. Drain. Div. Am. Soc. Civ. Eng., 103(IR3), 309-323, $1977 a$.

Katopodes, N. D., and T. Strelkoff, Dimensionless solutions of border irrigation advance, J. Irrig. Drain. Div. Am. Soc. Civ. Eng., 103(IR4), 401-417, $1977 b$.

Kincaid, D. C., Hydrodynamics of border irrigation, Ph.D. dissertation, Colo. State Univ., Fort Collins, 1970.

Sherman, B., A free boundary problem arising in a kinematic wave model of channel flow with infiltration, Q. Appl. Math., 39, 87-96, 1981.

Sherman, B., and V. P. Singh, A kinematic model for surface irrigation, Water Resour. Res., 14(2), 357-364, 1978.

Sherman, B., and V. P. Singh, Correction to 'A kinematic model for surface irrigation' by B. Sherman and V. P. Singh, Water Resour. Res., this issue.

Singh, V. P., and R. C. McCann, Mathematical modeling of hydraulics of irrigation recession, paper presented at the 2nd International Conference on Mathematical Modeling, Int. Assoc. Math. Model., St. Louis, Mo., July 11-13, 1979.

Streeter, V. L., and E. B. Wylie, Hydraulic Transients, McGrawHill, New York, 1967.

Strelkoff, T., and N. D. Katopodes, Border irrigation hydraulics with zero-inertia, J. Irrig. Drain. Div. Am. Soc. Civ. Eng., 103(IR3), 325-342, 1977a.

Strelkoff, T., and N. Katopodes, End depth under zero inertia conditions, J. Hydraul. Div. Am. Soc. Civ. Eng., 103(HY7), 699$711,1977 b$.

Stoker, J. J. Water Waves, Interscience, New York, 1957.

Wu, I. P., Recession flow in surface irrigation, J. Irrig. Drain. Div. Am. Soc. Civ. Eng., 98(IR1), 77-90, 1972.

(Received July 24, 1980; revised January 20, 1982; accepted February 8, 1982.) 